\title{
miR-21 promotes osteoclastogenesis through activation of PI3K/Akt signaling by targeting Pten in RAW264.7 cells
}

\author{
SHENGLI WANG ${ }^{1}$, ZHIGANG LIU ${ }^{1}$, JINGCHUN WANG ${ }^{2}$, XINYING JI ${ }^{3}$, ZHENQIANG YAO ${ }^{4}$ and XINCHUN WANG ${ }^{4}$ \\ Departments of ${ }^{1}$ Orthopedics and ${ }^{2}$ Pharmacy, The First Affiliated Hospital of Henan University; \\ ${ }^{3}$ Molecular Immunology Laboratory, Basic Medical College of Henan University; ${ }^{4}$ Molecular Biology Laboratory, \\ The First Affiliated Hospital of Henan University, Kaifeng, Henan 475001, P.R. China
}

Received June 4, 2019; Accepted November 18, 2019

DOI: $10.3892 / \mathrm{mmr} .2020 .10938$

\begin{abstract}
The present study aimed to investigate the effects of microRNA (miR)-21 on osteoclastogenesis and its underlying molecular mechanisms. The expression levels of tartrate-resistant acid phosphatase (TRAP) and miR-21 were detected during osteoclastogenesis in receptor activator of NF- $\kappa$ B ligand (RANKL)-induced RAW264.7 cells via reverse transcription-quantitative PCR. Bioinformatics and dual luciferase reporter assays were performed to analyze the association between miR-21 and Pten. RANKL-induced RAW264.7 cells were divided into the following groups: MiR-negative control (NC), miR-21 mimic, miR-21 inhibitor and miR-21 mimic + LY294002. The effects of miR-21 on osteoclastogenesis and bone resorption were then detected using TRAP staining and a bone resorption assay. Pten, phosphorylated-Akt and nuclear factor of activated $\mathrm{T}$ cell (NFATc1) expression levels were measured by western blotting to analyze the effects of miR-21 on the PI3K/Akt signaling pathway. The present study revealed that miR-21 was upregulated during osteoclastogenesis in RANKL-induced RAW264.7 cells. Furthermore, miR-21 negatively regulated Pten. Compared with the miR-negative control (NC) group, the number of osteoclasts and the percentage of bone resorption were increased in the miR-21 mimic group, whereas they were decreased in the miR-21 inhibitor group. The number of osteoclasts and the percentage of bone resorption in the miR-21 mimic + LY294002 group were lower than in the miR-21 mimic group. Compared with the miR-NC group, the protein expression levels of Pten were decreased, whereas p-Akt and NFATc1 were increased in the miR-21 mimic group. Conversely, Pten protein expression was increased, whereas p-Akt and NFATc1 were decreased in the miR-21 inhibitor group. In the miR-21 mimic + LY294002 group, Pten
\end{abstract}

Correspondence to: Dr Xinchun Wang, Molecular Biology Laboratory, The First Affiliated Hospital of Henan University, 357 Ximen Street, Kaifeng, Henan 475001, P.R. China

E-mail: researchone123@163.com

Key words: osteoclast, microRNA-21, Pten, PI3K/Akt protein expression was higher, and p-Akt and NFATc1 were lower than in the miR-21 mimic group. In conclusion, miR-21 is upregulated during osteoclastogenesis, and may promote osteoclastogenesis and bone resorption through activating the PI3K/Akt signaling pathway via targeting Pten.

\section{Introduction}

Osteoclasts (OCs) are primarily derived from mononuclear macrophages that arise from pluripotent hematopoietic stem cells; as the only cell with bone resorption function, OCs coordinate the dynamic balance between bone resorption and osteoblast formation (1). Numerous cytokines are involved in osteoclastogenesis from OC precursors. Macrophage colony-stimulating factor (M-CSF) and receptor activator of $\mathrm{NF}-\kappa \mathrm{B}$ ligand (RANKL) are produced by activated T cells and osteoblasts, and are essential cytokines for osteoclastogenesis (2). M-CSF regulates the proliferation and differentiation of mononuclear macrophages, binding to the cell surface expression receptor, c-Fms, whereas RANKL binds to the OC membrane receptor RANK and induces the expression of downstream signaling molecules, such as nuclear factor of activated T-cells cytoplasmic (NFATc1), NF- $\kappa \mathrm{B}$ and c-Fos, in order to regulate osteoclastogenesis $(3,4)$.

Numerous studies have reported that microRNAs (miRNAs/miRs) serve important roles during the process of osteoclastogenesis, including miR-503 (5), miR-148 (6) and miR-3 $(7,8)$, and are involved in this process via different signaling pathways. As an oncogene, miR-21 promotes the occurrence and progression of multiple myeloma and other solid tumors by inhibiting the expression of numerous tumor suppressor genes (9). Notably, the role of miR-21 in bone metabolism is gaining an increasing amount of interest. miR-21 inhibitor regulates the RANKL/osteoprotegerin (OPG) balance to decrease the bone resorption of mature OCs (10), and estrogen can downregulate miR-21, and upregulate Fas ligand (FasL) expression, inducing osteoclastic apoptosis (11). Therefore, it was hypothesized that miR-21 may be an important regulator associated with osteoclastogenesis and bone resorption. However, the molecular mechanism underlying the effects of miR-21 on OCs remains unclear.

In the present study, bioinformatics analysis predicted that Pten was a target gene of miR-21. Pten is a negative regulator 
of PI3K; Pten dephosphorylates PI3K, resulting in reduced PI3K/Akt signaling activity (12). Previous research has indicated that the PI3K/Akt signaling pathway has an important role in OC differentiation (13). Therefore, the present study aimed to investigate the effects of miR-21 on osteoclastogenesis and bone resorption, as well as the potential molecular mechanisms underlying the Pten-PI3K/Akt signaling pathway in OCs.

\section{Materials and methods}

Cell culture. The murine macrophage cell line RAW264.7 was purchased from the American Type Culture Collection and was cultured in DMEM (Gibco; Thermo Fisher Scientific, Inc.) supplemented with $10 \%$ fetal bovine serum (Gibco; Thermo Fisher Scientific, Inc.) and $1 \%$ penicillin $(100 \mathrm{U} / \mathrm{ml}) /$ streptomycin $(100 \mu \mathrm{g} / \mathrm{ml})$ (Hyclone; GE Healthcare Life Sciences) in an incubator containing $5 \% \mathrm{CO}_{2}$ at $37^{\circ} \mathrm{C}$. RAW264.7 cells were cultured with $50 \mathrm{ng} / \mathrm{ml}$ RANKL (Thermo Fisher Scientific, Inc.) for 3-5 days to induce osteoclastogenesis.

Dual-luciferase reporter gene assay. TargetScan (version 7.2; www.targetscan.org/vert_72) and miRTarBase (version 7.0; mirtarbase.mbc.nctu.edu.tw/php/index.php) were used to predict the potential target genes of miR-21. Wild-type (WT) and mutant (MT) binding sites of miR-21 in the 3'-untranslated region (UTR) of Pten were subcloned into pGL3 basic vectors (Promega Corporation), to generate pGL3-Pten-WT and pGL3-Pten-MT,respectively.RAW264.7 cells ( $1 \times 10^{6}$ cells/well) were seeded into 6-well plates and were transfected with $10 \mathrm{ng}$ Renilla luciferase plasmid (pRL-TK; Promega Corporation), $10 \mathrm{ng}$ pGL3-Pten-WT or pGL3-Pten-MT, and $25 \mu \mathrm{M}$ miR-21 mimic (Guangzhou RiboBio Co., Ltd.) or miR-negative control (NC; 5'-CAGUACUUUUGUGUAGUACAA-3'; Guangzhou RiboBio Co., Ltd.) using Lipofectamine ${ }^{\circledR}$ RNAi Max (Invitrogen; Thermo Fisher Scientific, Inc.), according to the manufacturer's protocol. After transfection, the cell were cultured at $37^{\circ} \mathrm{C}$ with $5 \% \mathrm{CO}_{2}$ for $48 \mathrm{~h}$, a Dual-Luciferase Reporter Gene Analysis system (Promega Corporation) was used to detect the luciferase activity, according to the manufacturer's protocol. With Renilla luciferase used as an internal control, luciferase activity was calculated as firefly fluorescence/Renilla fluorescence ratio.

Cell transfection. miR-NC (5'-CAGUACUUUUGUGUAGUA CAA-3), miR-21 mimic and miR-21 inhibitor (5'-UCAACA UCAGUCUGAUAAGCUA-3') were synthesized and obtained from Guangzhou RiboBio Co., Ltd. RAW264.7 cells at the logarithmic phase were seeded into 6-well plates and separated into the following groups: MiR-NC (cells transfected with $25 \mu \mathrm{M}$ miR-NC), miR-21 mimic (cells transfected with $25 \mu \mathrm{M}$ miR-21 mimic), miR-21 inhibitor (cells transfected with $25 \mu \mathrm{M}$ miR-21 inhibitor) and miR-21 mimic + LY294002 [cells transfected with miR-21 mimic and subsequently treated with the PI3K inhibitor LY294002 $(10 \mu \mathrm{M}$; APExBio Technology) and incubated at $37^{\circ} \mathrm{C}$ for $48 \mathrm{~h}$. At $70 \%$ confluence, the cells were transfected using Lipofectamine ${ }^{\circledR} 2000$ (Invitrogen; Thermo Fisher Scientific, Inc.), according to the manufacturer's protocol. At $48 \mathrm{~h}$ post-transfection at $37^{\circ} \mathrm{C}$ with $5 \% \mathrm{CO}_{2},>60 \%$ cells were successfully transfected.
Prior to subsequent reverse transcription-quantitative PCR (RT-qPCR), TRAP staining and western blotting experiments, the cells were cultured with $50 \mathrm{ng} / \mathrm{ml}$ RANKL for 3 days at $37^{\circ} \mathrm{C}$ with $5 \% \mathrm{CO}_{2}$ to induce osteoclastogenesis.

$R T-q P C R$. Total RNA was extracted from cells using TRIzol ${ }^{\circledR}$ reagent (Invitrogen; Thermo Fisher Scientific, Inc.), according to the manufacturer's protocol and was reverse transcribed using the SuperScript First Strand cDNA system (Invitrogen; Thermo Fisher Scientific, Inc.), according to the manufacturer's protocol. PCR amplification was performed using the SYBR Green PCR master mix (Thermo Fisher Scientific, Inc.). Stem-loop RT-qPCR and conventional RT-qPCR were used for quantification of miR-21, and tartrate-resistant acid phosphatase (TRAP; a specific marker of OCs and bone resorption) and Pten, respectively. The primers used in the present study were synthesized by Sangon Biotech Co., Ltd. (Table I). The reaction conditions were as follows: $95^{\circ} \mathrm{C}$ for $10 \mathrm{~min} ; 40$ cycles of $95^{\circ} \mathrm{C}$ for $15 \mathrm{sec}$ and $58^{\circ} \mathrm{C}$ for $30 \mathrm{sec} ; 72^{\circ} \mathrm{C}$ for $40 \mathrm{sec}$; and final extension at $72^{\circ} \mathrm{C}$ for $8 \mathrm{~min}$. The $2^{-\Delta \Delta \mathrm{C} q}$ method (14) was used to calculate the relative expression levels of miR-21, TRAP and Pten. miRNA and mRNA levels were normalized to the internal reference genes U6 and GAPDH, respectively.

TRAP staining. RANKL-induced RAW264.7 cells were seeded at a density of $5 \times 10^{5}$ cells/well into 24 -well plates and cultured for $24 \mathrm{~h}$ at $37^{\circ} \mathrm{C}$ with $5 \% \mathrm{CO}_{2}$. Subsequently, the cells were fixed with $4 \%$ paraformaldehyde for $20 \mathrm{~min}$ at room temperature. TRAP staining solution was added and incubated at $37^{\circ} \mathrm{C}$ for $1 \mathrm{~h}$ in the dark. The procedure was performed according to the TRAP staining kit protocol (Sigma Aldrich; Merck KGaA). TRAP-positive multi-nucleated cells (TRAP ${ }^{+}$ MNCs) containing $>3$ nuclei were considered OCs and were observed under an inverted light microscope (magnification, x200). The number of TRAP ${ }^{+}$MNCs/well was independently counted under the inverted microscope by two individuals. The average of the three experiments was taken as the number of OCs present.

Bone resorption assay. A pit assay was performed to observe the bone resorption of OCs. Bovine bone slices were generated in the laboratory and were obtained from fresh bovine cortical bone. Bone was purchased from cows that were sacrificed for commercial/consumer purposes. Notably, cows are not an endangered species, and the bones were obtained from a licensed source and did not contain potentially harmful agents (biological and chemical or genetically modified material). Bovine cortical bone was dried, rough-cut and sliced using a low speed diamond saw into pieces $\sim 50 \mu \mathrm{m}$ thick and $6 \mathrm{~mm}$ in diameter. Subsequently, the bone slices were treated in distilled water for ultrasonic cleaning prior to use. At $48 \mathrm{~h}$ post-transfection and prior to treatment with RANKL, RAW264.7 cells were seeded onto the bovine bone slices in 24-well plates at $5 \times 10^{5}$ cells/well and were cultured in routine medium for $24 \mathrm{~h}$ at $37^{\circ} \mathrm{C}$ with $5 \% \mathrm{CO}_{2}$. Subsequently, the medium was changed to routine medium supplemented with $50 \mathrm{ng} / \mathrm{ml}$ RANKL for 3 days, and then the bovine bone slices were ultrasonicated at $30 \mathrm{~Hz}$ for $10 \mathrm{~min}$ at room temperature in $1 \mathrm{~mol} / 1 \mathrm{NH}_{4} \mathrm{OH}$ to remove adherent cells. Bone slices 
Table I. Primers sequence for reverse transcription-quantitative PCR.

\begin{tabular}{|c|c|c|}
\hline \multirow[b]{2}{*}{ Gene } & \multicolumn{2}{|c|}{ Sequence $\left(5^{\prime} \rightarrow 3^{\prime}\right)$} \\
\hline & Forward & Reverse \\
\hline $\mathrm{miR}-21$ & 5'-CTCGCTTCGGCAGCACA-3' & 5'-GCCGCTAGCTTATCAGACTCAACA-3' \\
\hline U6 & 5'-CTCGCTTCGGCAGCACA-3' & 5'-AACGCTTCACGAATTTGCGT-3' \\
\hline TRAP & 5'-ACACAGTGATGCTGTGTGGCAACTC-3' & 5'-CCAGAGGCTTCCACATATATGATGG-3' \\
\hline Pten & 5'-AATTCCCAGTCAGAGGCGCTATGT-3' & 5'-GATTGCAAGTTCCGCCACTGAACA-3' \\
\hline GAPDH & 5'-AACATCAAATGGGGTGAGGCC-3' & 5'-GTTGTCATGGATGACCTTGGC-3' \\
\hline
\end{tabular}

miR, microRNA; TRAP, tartrate-resistant acid phosphatase.

were stained with $0.1 \%$ toluidine blue at room temperature for 3-5 min and the bone resorption pits were subsequently observed under a light microscope (magnification, $\mathrm{x} 400$ ). Bone resorption was calculated as pit area/total bone area of each slice, and was calculated using Image J software (version 1.8.0; National Institutes of Health).

Western blot analysis. Total protein was extracted from cells using a Total Protein Extraction kit (Beiing Solarbio Science and Technology Co., Ltd.), according to the manufacturer's protocol. The bicinchoninic acid method was used to measure protein concentration and $40 \mu \mathrm{g}$ protein/lane was separated by SDS-PAGE on $10 \%$ gels and transferred to PVDF membranes (EMD Millipore). PVDF membranes were blocked in TBS containing $0.25 \%$ Tween and $5 \%$ skimmed milk for $1 \mathrm{~h}$ at room temperature. Membranes were incubated with primary antibodies targeted against: Pten (cat. no. ab32199; 1:1,000), Akt (cat. no. ab18785; 1:5,000), phosphorylated (p)-Akt (cat. no. ab38449; 1:1,000), NFATc1 (cat. no. ab25916; 1:500) and GAPDH (cat. no. ab9485; 1:5,000) at $4^{\circ} \mathrm{C}$ overnight. All primary antibodies were purchased from Abcam. Membranes were then incubated with horseradish peroxidase-conjugated IgG secondary antibodies (cat. no. ab6721; 1:10,000; Abcam) for $1 \mathrm{~h}$ at room temperature. ECL detection reagent (Beiing Solarbio Science and Technology Co., Ltd.) was used to observe protein bands and relative protein expression levels were calculated using ImageJ software (version 1.8.0; National Institutes of Health) with GAPDH as an internal reference.

Statistical analysis. Each experiment was repeated at least 3 times. SPSS software (version 19.0; IBM Corp.) was used to analyze all data. Kolmogorov-Smirnov test was used to confirm that data were normally distributed and data are presented as the mean \pm SD. Comparisons among multiple groups were assessed by one-way ANOVA followed by Tukey's post hoc test. An unpaired Student's t-test was used to assess the differences between the two groups in the dual-luciferase assay. $\mathrm{P}<0.05$ was considered to indicate a statistically significant difference.

\section{Results}

Expression of miR-21 is upregulated during osteoclastogenesis. The mRNA expression levels of the OC marker TRAP were detected in RANKL-induced macrophage RAW264.7 cells at different time points by RT-qPCR, in order to analyze osteoclastogenesis (Fig. 1A). After 3 days of induction, the expression levels of TRAP were significantly increased, and the expression of TRAP was significantly higher on the fifth day compared with on the first and third days. Our preliminary studies detected the background levels of TRAP expression at $0 \mathrm{~h}$ and confirmed that they are lower than at the first day of RANKL induction; therefore, the expression of TRAP at $0 \mathrm{~h}$ was not recorded in the present study. As presented in Fig. 1B, the expression levels of miR-21 increased progressively during the process of osteoclastogenesis and the change in miR-21 was consistent with the upregulation of TRAP mRNA, indicating that miR-21 was upregulated during osteoclastogenesis in vitro.

miR-21 targets Pten. Pten promotes the proliferation of T cells and tumor cells, and has a critical role in RANKL-induced OC differentiation $(15,16)$. In the present study, target genes that were potentially regulated by miR-21 were predicted by TargetScan and miRTarBase; Pten, which contains miR-21 binding sites in its 3'UTR, was selected as one of the target genes of miR-21. The results of a dual luciferase reporter gene assay revealed that the luciferase activity of Pten-WT was inhibited by the miR-21 mimic $(\mathrm{P}<0.05)$, whereas the luciferase activity of Pten-MT was not affected, thus suggesting that miR-21 directly targeted Pten (Fig. 2A).

In order to verify the effects of miR-21 on Pten, the expression levels of miR-21 and Pten were detected in RANKL-induced RAW264.7 cells post-transfection by RT-qPCR and western blotting (Fig. 2B-D). Compared with the miR-NC group, miR-21 expression was increased, whereas Pten protein expression was decreased in the miR-21 mimic group. Furthermore, the expression levels of miR-21 were downregulated, whereas the protein expression levels of Pten were upregulated in the miR-21 inhibitor group. There was no difference in Pten mRNA expression among the groups. These results suggested that miR-21 negatively regulated Pten at the translational level.

miR-21 promotes osteoclastogenesis. TRAP staining was used to detect osteoclastogenesis of RAW264.7 cells transfected with miR-21 mimic and miR-21 inhibitor, in order to assess the effect of miR-21 on OC differentiation (Fig. 3). 

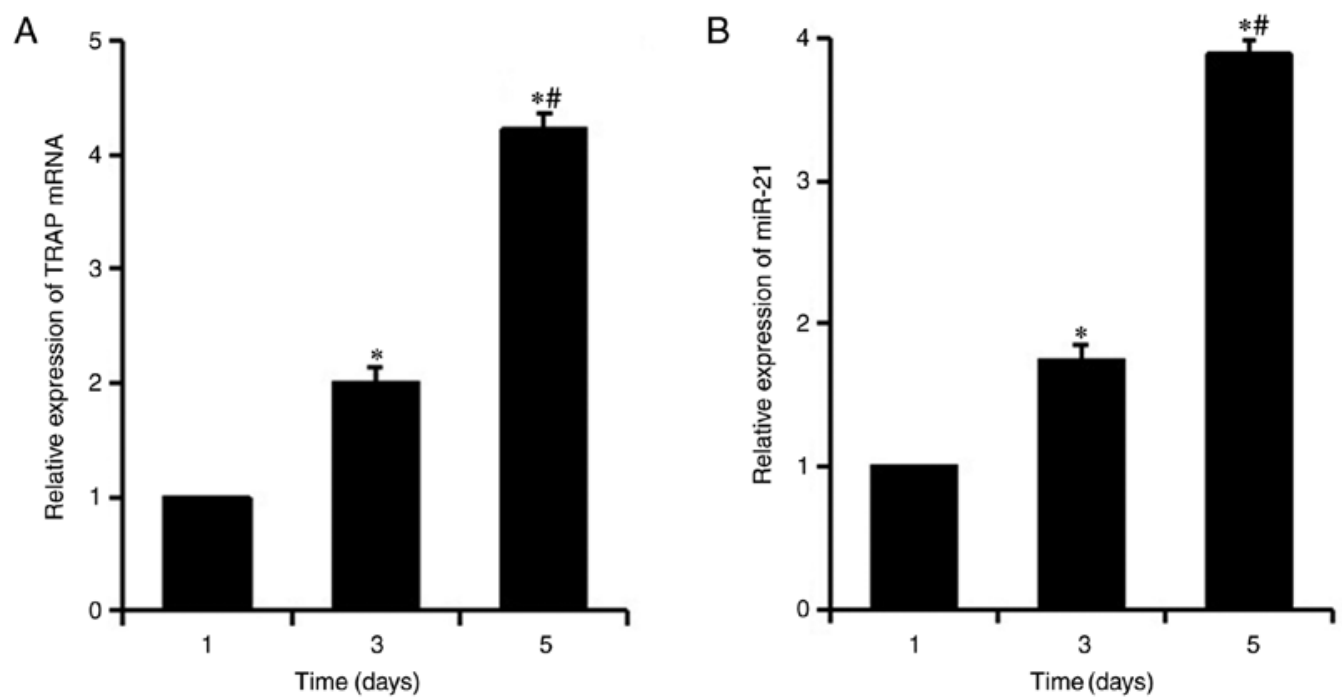

Figure 1. Expression of miR-21 and TRAP during osteoclastogenesis. Relative expression levels of (A) TRAP and (B) miR-21 in RANKL-induced RAW264.7 cells. ${ }^{*} \mathrm{P}<0.05$ vs. day $1 ;{ }^{*} \mathrm{P}<0.05$ vs. day 3 . miR-21, microRNA-21; RANKL, receptor activator of NF- $\kappa$ B ligand; TRAP, tartrate-resistant acid phosphatase.

A

miR-21: $\quad$ 3'-agUUGUAGUCAGACUAUUCGAu-5'

\|\|||$\||| \mid$

Pten: 5'-uaGUCAUCACU--UAAUAAGUUa-3'
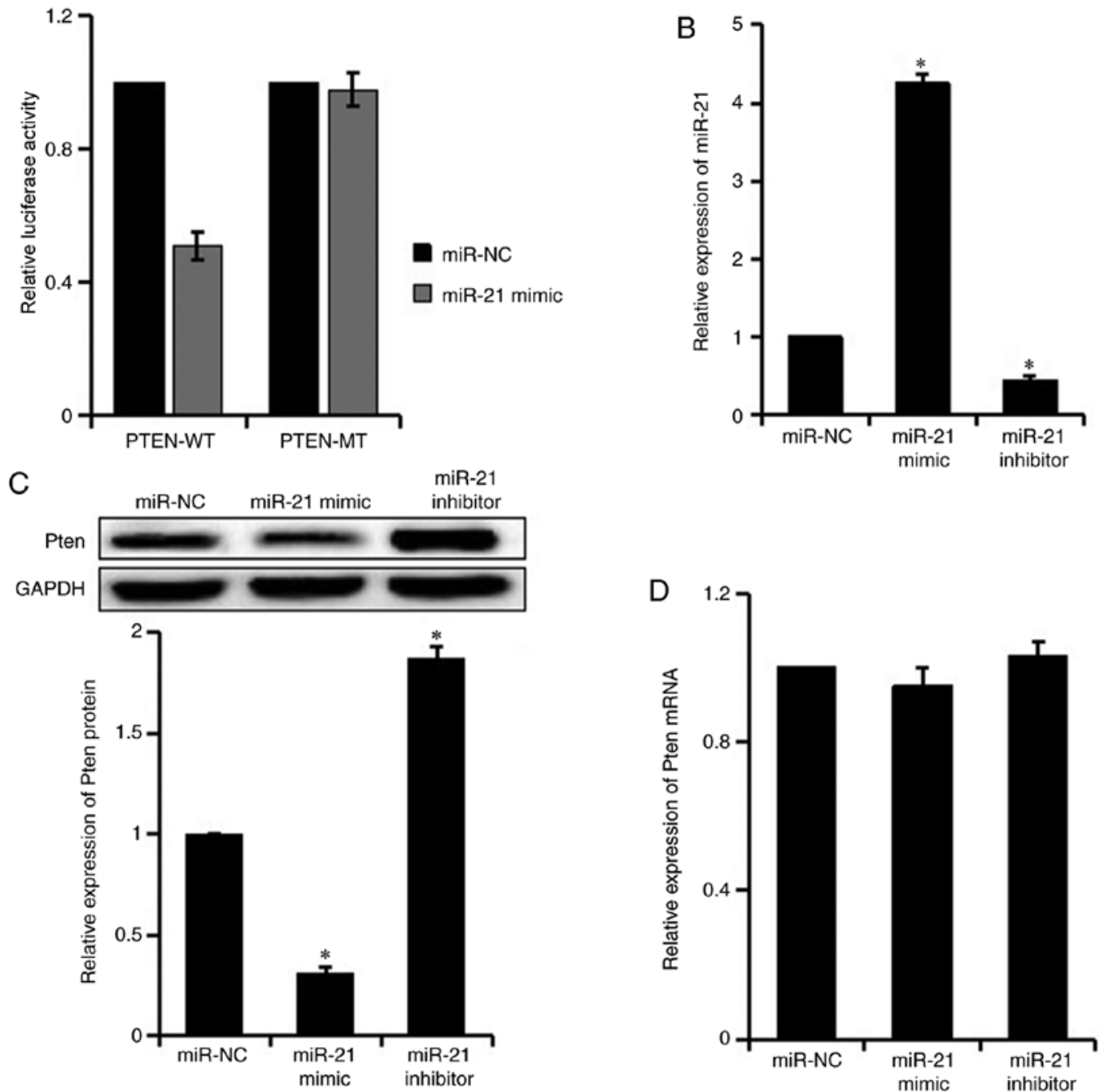

Figure 2. miR-21 targets Pten. (A) Sequence alignment of miR-21 with the 3'-untranslated region of Pten and results of a dual luciferase reporter gene assay. Relative expression levels of (B) miR-21, (C) Pten protein and (D) Pten mRNA post-transfection. "P<0.05 vs. miR-NC. miR, microRNA; MT, mutant; NC, negative control; WT, wild type. 

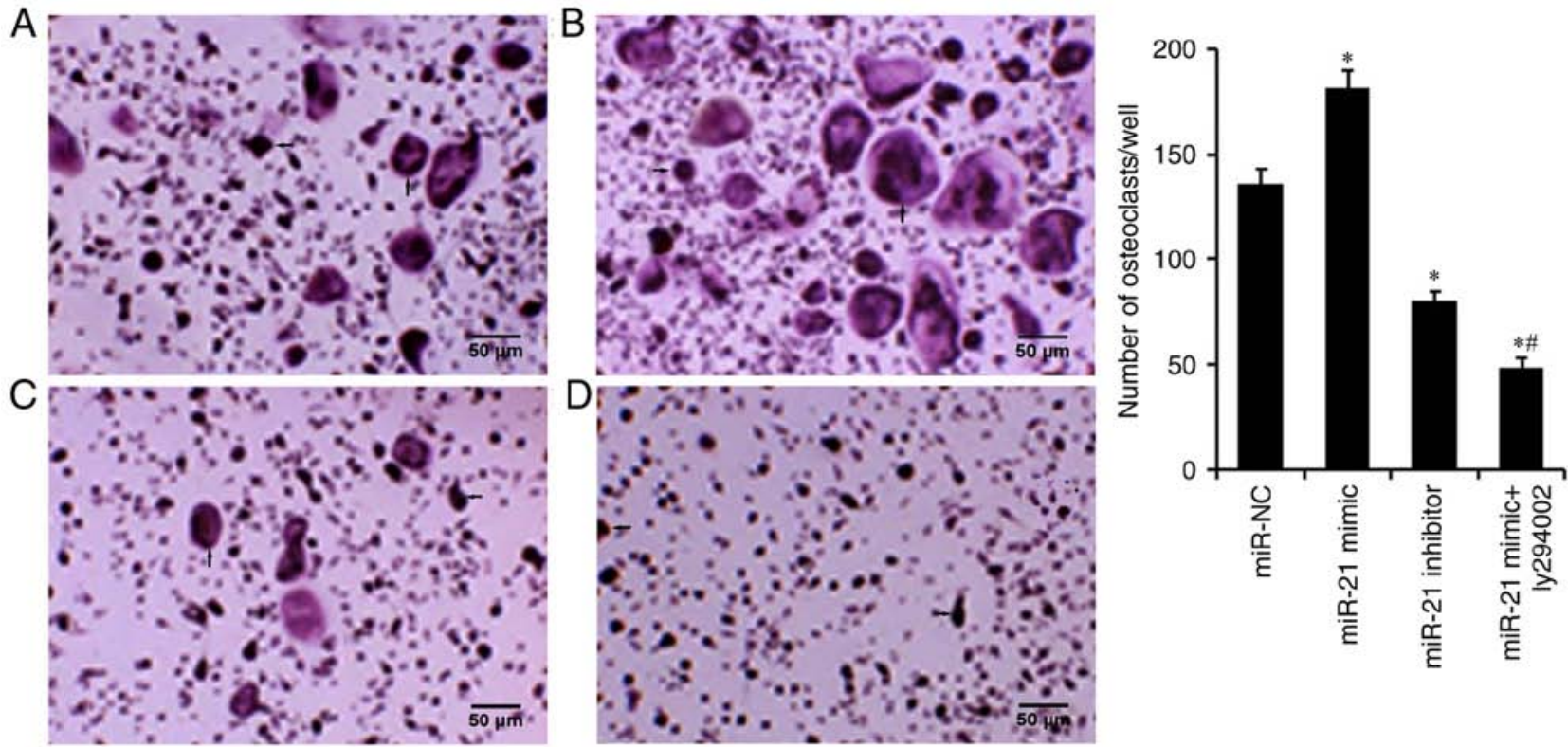

Figure 3. TRAP staining and number of osteoclasts. (A) miR-NC group, (B) miR-21 mimic group, (C) miR-21 inhibitor group and (D) miR-21 mimic + LY294002 group. Arrows indicate osteoclasts (TRAP ${ }^{+}$multi-nucleated cells). ${ }^{*} \mathrm{P}<0.05$ vs. miR-NC; ${ }^{*} \mathrm{P}<0.05$ vs. miR-21 mimic. Scale bars, $50 \mu \mathrm{m}$. miR, microRNA; NC, negative control; TRAP, tartrate resistant acid phosphatase.
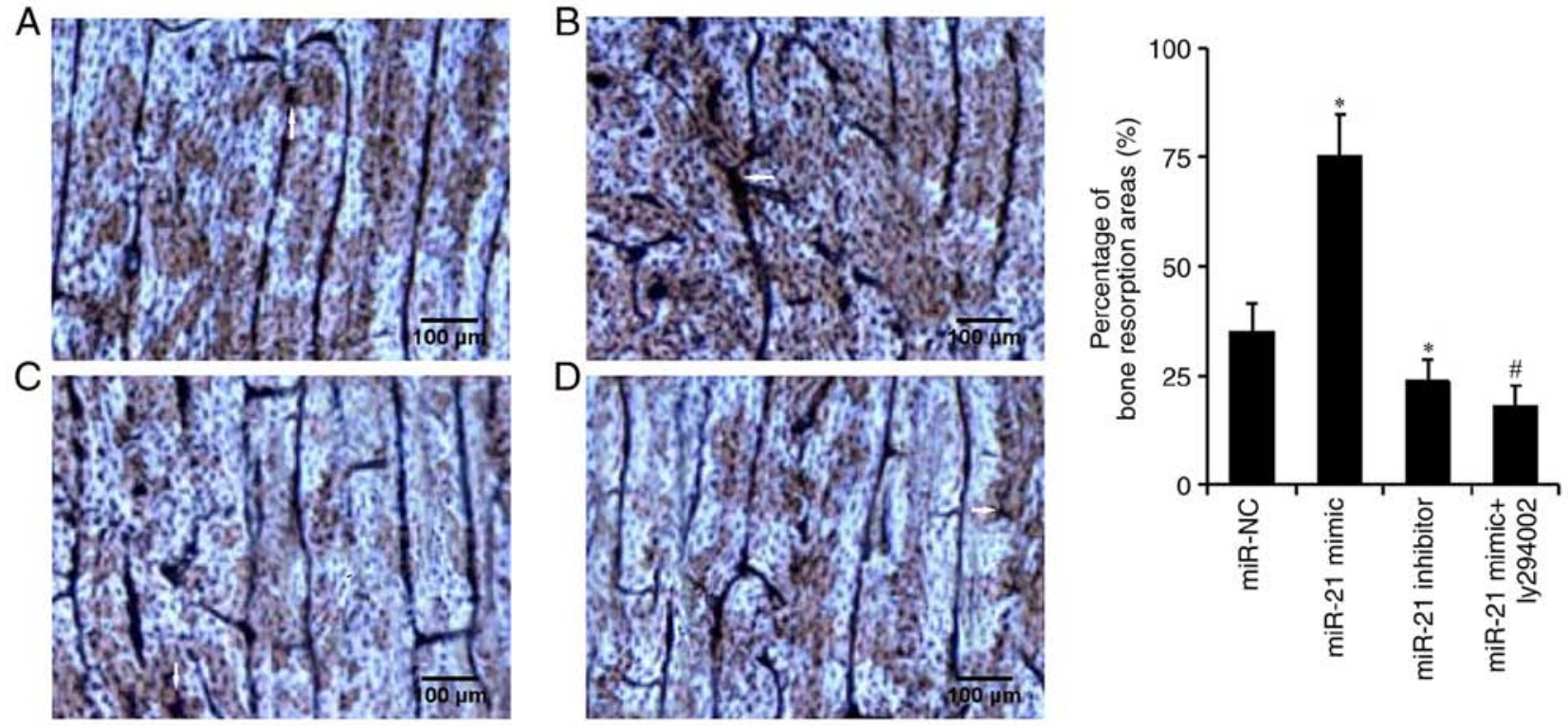

Figure 4. Bone resorption areas on bovine bone slices. (A) miR-NC group, (B) miR-21 mimic group, (C) miR-21 inhibitor group and (D) miR-21 mimic + LY294002 group. Arrows indicate bone resorption areas. " $\mathrm{P}<0.05$ vs. miR-NC; ${ }^{*} \mathrm{P}<0.05$ vs. miR-21 mimic. Scale bars, $100 \mu \mathrm{m}$. miR, microRNA; NC, negative control.

The results revealed that the number of OCs was increased in the miR-21 mimic group and markedly decreased in the miR-21 inhibitor group, when compared with the miR-NC group, thus indicating that miR-21 may be crucial for osteoclastogenesis.

miR-21 enhances bone resorption of OCs. The bone resorption assay was used to detect the percentage of bone resorption (Fig. 4). The percentage of bone resorption was significantly higher in the miR-21 mimic group than in the miR-NC group $(75.4 \pm 10.2$ vs. $35.1 \pm 7.3 \%$, respectively), and was significantly higher than that in the miR-21 inhibitor group $(23.8 \pm 5.6 \%)$. These results suggested that miR-21 could enhance the bone resorption of OCs.

miR-21 promotes osteoclastogenesis and bone resorption via regulation of the Pten-PI3K/Akt signaling pathway. It has previously been indicated that Pten regulates osteoclastogenesis in RANKL-induced RAW264.7 OC precursors via activating the PI3K/Akt signaling pathway (16). In the present study, the results of TRAP staining and the bone resorption assay revealed that the number of OCs and the percentage of 
bone resorption in the miR-21mimic + LY294002 group were lower than those in the miR-21 mimic group (Figs. 3 and 4). These findings indicated that miR-21 may regulate osteoclastogenesis and bone resorption via regulating the PI3K/Akt signaling pathway. The present study further detected Pten, Akt, p-Akt and NFATc1 expression in RAW264.7 cells post-transfection, as presented in Fig. 5. The results demonstrated that, compared with the miR-NC group, the protein expression levels of Pten were decreased, and p-Akt and NFATc1 expression was increased in the miR-21 mimic group. Conversely, the protein expression levels of Pten were increased, whereas p-Akt and NFATc1 expression levels were decreased in the miR-21 inhibitor group. Compared with the miR-21 mimic group, the protein expression levels of Pten were upregulated, whereas p-Akt and NFATc1 expression were reduced in the miR-21 mimic + LY294002 group, thus suggesting that the PI3K inhibitor LY294002 strongly reversed the effects induced by the miR-21 mimic. These results indicated that miR-21 may promote osteoclastogenesis and bone resorption via activation of the PI3K/Akt signaling pathway by targeting Pten.

\section{Discussion}

Kagiya and Nakamura (17) reported that $>50$ miRNAs, including miR-21, miR-146a and miR-155, are abnormally expressed during TNF- $\alpha /$ RANKL-regulated osteoclastogenesis, thus suggesting that these miRNAs may be essential in the regulation of OC differentiation. Emerging evidence has indicated that miRNAs serve important roles in osteoblastogenesis, chondrocyte proliferation and differentiation, and osteoclastogenesis (18). However, the role of miRNAs in the process of OC differentiation and its underlying molecular mechanism remain to be elucidated; identifying these roles may provide a novel direction for the clinical treatment of diseases associated with bone metabolism.

As a carcinogenic miRNA, miR-21 is upregulated in almost all types of cancer, and is responsible for promoting their occurrence and development, including gastric cancer (19), colorectal cancer (20) and non-small cell lung cancer (21). In recent years, the effect of miR-21 on bone metabolism has received extensive attention: Upregulation of miR-21 has been reported to not only increase the expression of osteopontin and alkaline phosphatase in osteogenesis, but may also promote mineralization during osteogenic induction (22). Furthermore, it was verified that miR-21 acts as a regulator of osteoclastogenesis and a promoter of $\mathrm{OC}$ differentiation in vitro and in vivo $(16,23)$. Xu et al (24) also confirmed that miR-21 was upregulated in A549 cells and overexpression of miR-21 facilitated osteoclastogenesis by increasing the levels of miR-21 in exosomes. In the present study, miR-21 was upregulated during osteoclastogenesis in RANKL-induced RAW264.7 cells, and it was revealed that upregulation of miR-21 could promote OC differentiation and bone resorption, whereas downregulation of miR-21 could inhibit OC differentiation and bone resorption. These findings indicated that miR-21 was crucial to osteoclastogenesis.

At present, the molecular mechanism underlying the effects of miR-21 on the regulation of osteoclastogenesis remains unclear. Sugatani et al (25) demonstrated that the
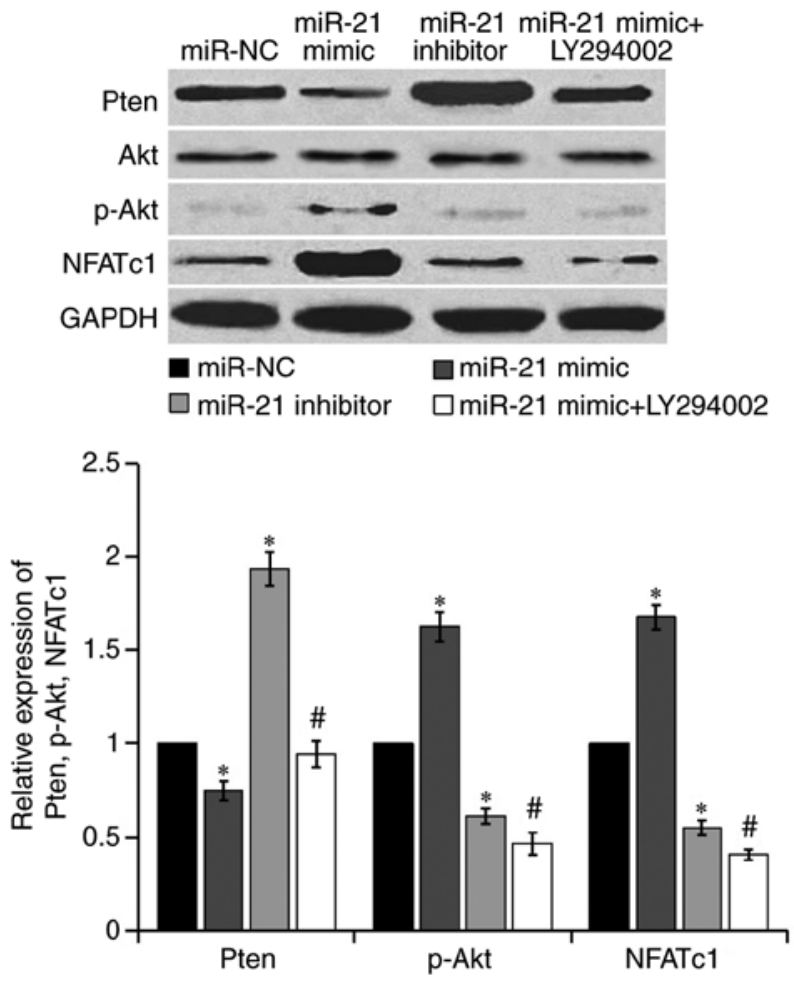

Figure 5. Relative protein expression levels of Pten, p-Akt, Akt and NFATc1. ${ }^{*} \mathrm{P}<0.05$ vs. miR-NC group; ${ }^{\#} \mathrm{P}<0.05$ vs. miR-21 mimic group. miR, microRNA; NC, negative control; NFATc1, nuclear factor of activated T-cell; p, phosphorylated.

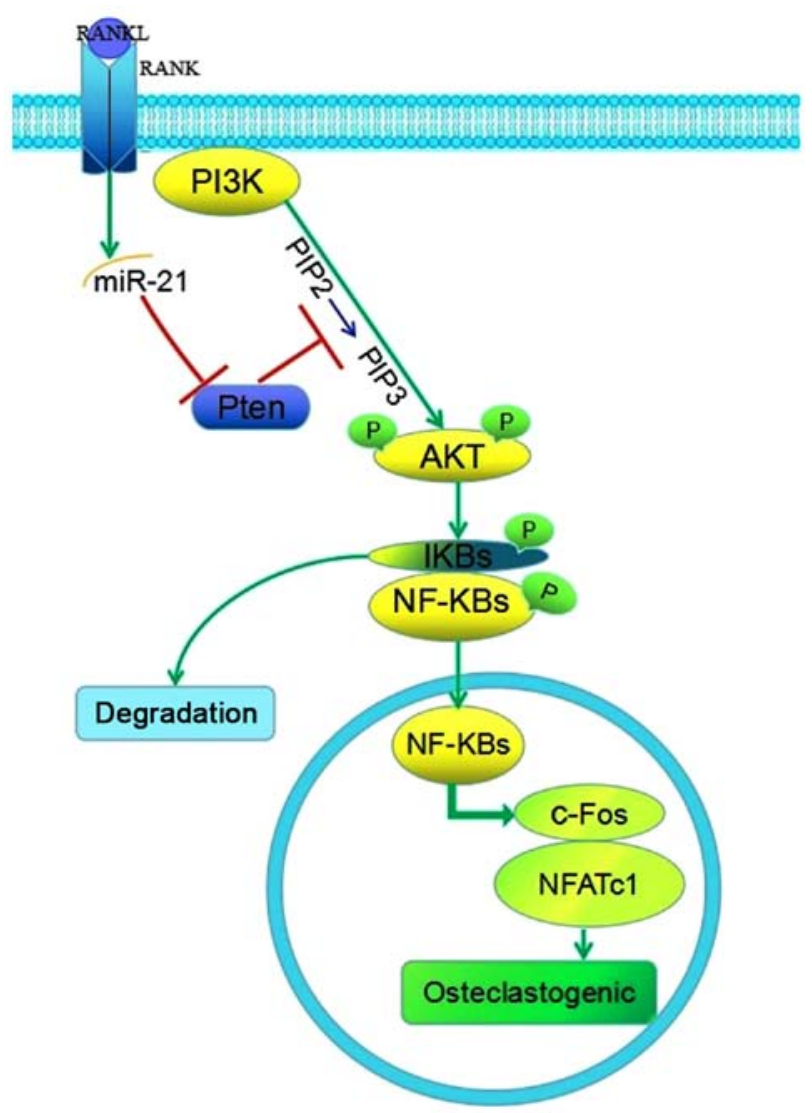

Figure 6. Effect of miR-21 on osteoclastogenesis. miR-21 regulated the PI3K/Akt signaling pathway in osteoclast differentiation by targeting Pten. miR, microRNA; RANK, receptor activator of NF- $\kappa$ B; RANKL, RANK

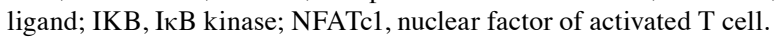


transcription factor c-Fos upregulated miR-21 expression, and miR-21 downregulated programmed cell death (PDCD4) protein expression, whereas diminished PDCD4 removed the suppressive effects of c-Fos in the process of RANKL-induced osteoclastogenesis; therefore, a positive feedback loop of c-Fos/miR-21/PDCD4 may be a potential novel molecular mechanism underlying the regulation of osteoclastogenesis. Fujita et al (26) also confirmed that c-Fos promoted OC differentiation by binding to miR-21 through activation protein 1 , and that miR-21 served a central role during estrogen-controlled osteoclastogenesis: Estrogen induced osteoclast apoptosis by downregulating miR-21 expression and by increasing the target gene of miR-21, FasL. Pitari et al (10) reported that miR-21 expression was markedly enhanced, whereas OPG was strongly reduced in bone marrow stromal cell (BMSCs) adherent multiple myeloma cells and OPG was targeted by miR-21. Furthermore, treatment of BMSCs with miR-21 inhibitors restored RANKL/OPG balance and markedly impaired the resorptive activity of mature OCs (10). However, a number of target genes of miR-21 have been revealed via bioinformatics analysis. In the present study, bioinformatics and dual luciferase reporter assays verified that Pten was a target gene of miR-21, and Pten was negatively regulated by miR-21. Pten is a tumor suppressor and as a dual-specificity phosphatase, it regulates the cell cycle, inhibits cell proliferation and promotes apoptosis by blocking the PI3K/Akt signaling pathway. Conversely, miR-21 promotes tumor cell growth, proliferation, invasion and metastasis by inhibiting Pten, which leads to abnormal activation of the PI3K/Akt pathway; this is particularly associated with an increase in p-Akt $(27,28)$. The present study demonstrated that the number of OCs and the percentage of bone resorption were decreased in the miR-21 mimic + LY294002 group compared with in the miR-21 mimic group. Compared with the miR-21 mimic group, the protein expression levels of Pten were increased, and p-Akt and NFATc1 expression was decreased in the miR-21 mimic + LY294002 group, indicating that LY294002 reversed the mir-21 mimic-induced decreases in Pten, and increases in p-Akt and NFATc1. It has therefore been hypothesized that miR-21 promoted osteoclastogenesis and bone resorption through activation of the PI3K/Akt signaling pathway by targeting Pten (Fig. 6).

In conclusion, the present study revealed that miR-21 was upregulated in osteoclastogenesis, and promoted osteoclastogenesis and bone resorption via activating the PI3K/Akt signaling pathway through targeting Pten. These results provided preliminary evidence towards elucidating the molecular mechanism underlying how miR-21 promotes osteoclastogenesis, laying a theoretical foundation for the clinical treatment of diseases associated with bone metabolism, including osteoporosis and osteomalacia.

\section{Acknowledgements}

Not applicable.

\section{Funding}

The present study was funded by the Natural Science Foundation of China (grant. no. 81471558).

\section{Availability of data and materials}

The datasets used and/or analyzed during the current study are available from the corresponding author on reasonable request, and in the TargetScan (www.targetscan.org/vert_72) and miRTarBase (mirtarbase.mbc.nctu.edu.tw/php/index.php) databases.

\section{Authors' contributions}

SW and XW designed the study. SW drafted the manuscript. ZL performed TRAP staining and the bone resorption assay. JW analyzed and verified the target gene of miR-21. XJ performed western blotting. ZY acquired, analyzed and interpreted the data. XW revised the manuscript. All authors approved the final published version of this manuscript.

\section{Ethics approval and consent to participate}

Not applicable.

\section{Patient consent for publication}

Not applicable.

\section{Competing interests}

The authors declare that they have no competing interests.

\section{References}

1. Drissi $\mathrm{H}$ and Sanjay A: The multifaceted osteoclast; far and beyond bone resorption. J Cell Biochem 117: 1753-1756, 2016.

2. Boyce BF: Advances in the regulation of osteoclasts and osteoclast functions. J Dent Res 92: 860-867, 2013.

3. Hodge JM, Collier FM, Pavlos NJ, Kirkland MA and Nicholson GC: M-CSF potently augments RANKL-induced resorption activation in mature human osteoclasts. PLoS One 6: e21462, 2011.

4. Takayanagi H, Kim S, Koga T, Nishina H, Isshiki M, Yoshida H, Saiura A, Isobe M, Yokochi T, Inoue J, et al: Induction and activation of the transcription factor NFATc1 (NFAT2) integrate RANKL signaling in terminal differentiation of osteoclasts. Dev Cell 3: 889-901, 2002.

5. Chen C, Cheng P, Xie H, Zhou HD, Wu XP, Liao EY and Luo XH: MiR-503 regulates osteoclastogenesis via targeting RANK. J Bone Miner Res 29: 338-347, 2014.

6. Cheng P, Chen C, He HB, Hu R, Zhou HD, Xie H, Zhu W, Dai RC, Wu XP, Liao EY and Luo XH: miR-148a regulates osteoclastogenesis by targeting V-maf musculoaponeurotic fibrosarcoma oncogene homolog B. J Bone Miner Res 28: 1180-1190, 2013.

7. Mizoguchi F, Murakami Y, Saito T, Miyasaka N and Kohsaka H: miR-31 controls osteoclast formation and bone resorption by targeting RhoA. Arthritis Res Ther 15: R102, 2013.

8. Ge J, Guo S, Fu Y, Zhou P, Zhang P, Du Y, Li M, Cheng J and Jiang H: Dental follicle cells participate in tooth eruption via the RUNX2-MiR-31-SATB2 loop. J Dent Res 94: 936-944, 2015.

9. Wang JH, Zheng WW, Cheng ST, Liu BX, Liu FR and Song JQ: Correlation between microRNA21 and sprouty homolog 2 gene expression in multiple myeloma. Mol Med Rep 11: 4220-4224, 2015.

10. Pitari MR, Rossi M, Amodio N, Botta C, Morelli E, Federico C, Gullà A, Caracciolo D, Di Martino MT, Arbitrio M, et al: Inhibition of miR-21 restores RANKL/OPG ratio in multiple myeloma-derived bone marrow stromal cells and impairs the resorbing activity of mature osteoclasts. Oncotarget 6: 27343-27358, 2015.

11. Sugatani T and Hruska KA: Down-regulation of miR-21 biogenesis by estrogen action contributes to osteoclastic apoptosis. J Cell Biochem 114: 1217-1222, 2013. 
12. Ocana A, Vera-Badillo F, Al-Mubarak M, Templeton AJ, Corrales-Sanchez V, Diez-Gonzalez L, Cuenca-Lopez MD, Seruga B, Pandiella A and Amir E: Activation of the $\mathrm{PI} 3 \mathrm{~K} / \mathrm{mTOR} / \mathrm{AKT}$ pathway and survival in solid tumors: Systematic review and meta-analysis. PLoS One 9: e95219, 2014

13. Wang $L$ and Guo X: Research advance in signal pathways and signal factors during the process of osteoclast differentiation. Chin J Osteoporo 21: 742-748, 2015.

14. Livak KJ and Schmittgen TD: Analysis of relative gene expression data using real-time quantitative PCR and the 2(-Delta Delta C(T)) method. Methods 25: 402-408, 2001.

15. Wan J, Ling X, Peng B and Ding G: MiR-142-5p regulates CD4+ $\mathrm{T}$ cells in human non-small cell lung cancer through PD-L1 expression via the PTEN pathway. Oncol Rep 40: 272-282, 2018.

16. Jang HD, Noh JY, Shin JH, Lin JJ and Lee SY: PTEN regulation by the Akt/GSK-3 $\beta$ axis during RANKL signaling. Bone 55: $126-131,2013$

17. Kagiya T and Nakamura S: Expression profiling of microRNAs in RAW264.7 cells treated with a combination of tumor necrosis factor alpha and RANKL during osteoclast differentiation. J Periodontal Res 48: 373-385, 2013.

18. Deng Z and Jin J: MicroRNAs in regulation of bone metabolism: A literature review. Orthop J China 24: 1019-1022, 2016.

19. Zhang BG, Li JF, Yu BQ, Zhu ZG, Liu BY and Yan M: microRNA-21 promotes tumor proliferation and invasion in gastric cancer by targeting PTEN. Oncol Rep 27: 1019-1026, 2012.

20. Peacock O, Lee AC, Cameron F, Tarbox R, Vafadar-Isfahani N, Tufarelli $\mathrm{C}$ and Lund JN: Inflammation and MiR-21 pathways functionally interact to downregulate PDCD4 in colorectal cancer. PLoS One 9: e110267, 2014

21. $\mathrm{Xu} \mathrm{LF}, \mathrm{Wu} \mathrm{ZP}$, Chen Y, Zhu QS, Hamidi S and Navab R: MicroRNA-21 (miR-21) regulates cellular proliferation, invasion, migration, and apoptosis by targeting PTEN, RECK and Bcl-2 in lung squamous carcinoma, Gejiu City, China. PLoS One 9: e103698, 2014.
22. Sun Y, Xu L, Huang S, Hou Y, Liu Y, Chan KM, Pan XH and Li G: mir-21 overexpressing mesenchymal stem cells accelerate fracture healing in a rat closed femur fracture model. Biomed Res Int 2015: 412327, 2015.

23. Hu CH, Sui BD, Du FY, Shuai Y, Zheng CX, Zhao P, Yu XR and Jin Y: miR-21 deficiency inhibits osteoclast function and prevents bone loss in mice. Sci Rep 7: 43191, 2017.

24. Xu Z, Liu X, Wang H, Li J, Dai L, Li J and Dong C: Lung adenocarcinoma cell-derived exosomal miR-21 facilitates osteoclastogenesis. Gene 666: 116-122, 2018.

25. Sugatani T, Vacher J and Hruska KA: A microRNA expression signature of osteoclastogenesis. Blood 117: 3648-3657, 2011.

26. Fujita S, Ito T Mizutani T, Minoguchi S, Yamamichi N, Sakurai K and Iba H: miR-21 gene expression triggered by AP-1 is sustained through a double-negative feedback mechanism. J Mol Biol 378: 492-504, 2008.

27. Wang P, Guan Q, Zhou D, Yu Z, Song Y and Qiu W: miR-21 inhibitors modulate biological functions of gastric cancer cells via PTEN/PI3K/mTOR pathway. Dna Cell Biol 37: 38-45, 2018.

28. Xiong B, Cheng Y, Ma L and Zhang C: MiR-21 regulates biological behavior through the PTEN/PI-3 K/Akt signaling pathway in human colorectal cancer cells. Int J Oncol 42: 219-228, 2013.

This work is licensed under a Creative Commons Attribution-NonCommercial-NoDerivatives 4.0 International (CC BY-NC-ND 4.0) License. 